\title{
Pressure Loss Predictions of the Reactor Simulator Subsystem at NASA GRC
}

\author{
Terry V. Reid ${ }^{1}$ \\ NASA Glenn Research Center, Cleveland, Ohio, 44135
}

\begin{abstract}
Testing of the Fission Power System (FPS) Technology Demonstration Unit (TDU) is being conducted at NASA GRC. The TDU consists of three subsystems: the Reactor Simulator (RxSim), the Stirling Power Conversion Unit (PCU), and the Heat Exchanger Manifold (HXM). An Annular Linear Induction Pump (ALIP) is used to drive the working fluid. A preliminary version of the TDU system (which excludes the PCU for now), is referred to as the RxSim subsystem and was used to conduct flow tests in Vacuum Facility 6 (VF 6). In parallel, a computational model of the RxSim subsystem was created based on the CAD model and was used to predict loop pressure losses over a range of mass flows. This was done to assess the ability of the pump to meet the design intent mass flow demand. Measured data indicates that the pump can produce $2.333 \mathrm{~kg} / \mathrm{sec}$ of flow, which is enough to supply the RxSim subsystem with a nominal flow of $1.75 \mathrm{~kg} / \mathrm{sec}$. Computational predictions indicated that the pump could provide $2.157 \mathrm{~kg} / \mathrm{sec}$ (using the Spalart-Allmaras turbulence model), and 2.223 $\mathrm{kg} / \mathrm{sec}$ (using the $\mathrm{k}-\varepsilon$ turbulence model). The computational error of the predictions for the available mass flow is $-\mathbf{0 . 1 7 6} \mathrm{kg} / \mathrm{sec}$ (with the $S-A$ turbulence model) and $\mathbf{- 0 . 1 1 0 ~} \mathrm{kg} / \mathrm{sec}$ (with the $k-\varepsilon$ turbulence model) when compared to measured data.
\end{abstract}

\section{Nomenclature}

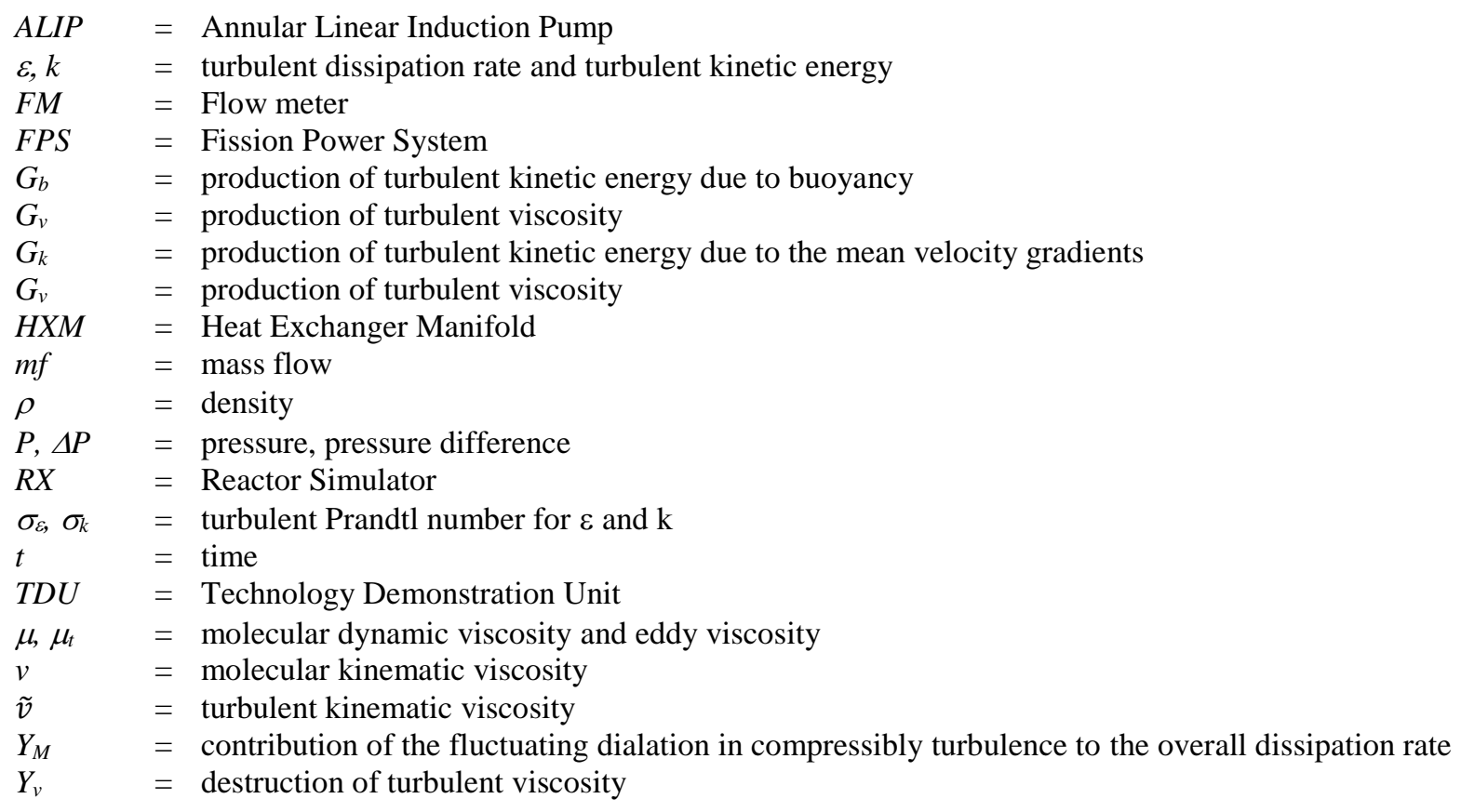

\section{Introduction}

G xploration of our solar system continues to bring many exciting challenges to our nation's scientific and Eengineering community. As we expand our visions to explore new, more challenging destinations, we must also

${ }^{1}$ Mechanical Engineer, Thermal Energy Conversion Branch, 21000 Brookpark Road/MS 301-2, member.

American Institute of Aeronautics and Astronautics 
expand our technology base to support these new missions. NASA's Space Technology and Mission Directorate (STMD) is tasked with defining and developing these technologies for future mission infusion and continues to seek answers to many existing technology gaps. One such technology gap is related to compact power systems $(>1 \mathrm{kWe})$ that do not rely on solar energy and can provide abundant power for several years if not decades. Below $1 \mathrm{kWe}$, Radioisotope Power Systems (RPS) have been the workhorse for NASA and will continue to be used for lower power applications similar to prior missions like Voyager, Ulysses, New Horizons, Cassini, and Curiosity. Above $1 \mathrm{kWe}$, Fission Power Systems (FPS) become the base technology with multiple options for reactor design, power conversion, and heat rejection which are driven by specific power needs and mission requirements.

At NASA, efforts are being made to develop a Technology Demonstration Unit (TDU) that uses Fission Power System technology to produce $\sim 12 \mathrm{~kW}$ of electric power. As components of the TDU are tested and developed, the components are then assembled for subsequent test, with lessons learned applied along the way. One of the main components of the FPS is a pump that circulates liquid-metal throughout the reactor system. This annular linear induction pump (ALIP) needs to meet specific mission requirements and is tested at representative operating conditions. In the early stages of testing (originally performed at NASA MSFC), testing was performed using the Reactor Simulator loop to verify that the selected ALIP could deliver the required pressure and flow at nominal conditions. While testing with the TDU ALIP in place, it was discovered that this particular pump could not deliver the required pressure and flow at nominal conditions. A FPS ALIP with a larger capacity than the TDU ALIP was available for use, but testing was needed to verify that FPS ALIP could provide the needed pressure and flow rate as a component in the Reactor Simulator loop. The Reactor Simulator loop was moved to NASA GRC with the intention of rebuilding the loop, and conducting tests using the FSP ALIP. Prior to the assembly of this loop, a computational model of the RxSim loop was constructed in an effort to generate predictions that tracked the pressure as a function of flow rate throughout the loop, and over the anticipated operating range of the system.

\section{Problem Statement}

In the Reactor Simulator subsystem, an Annular Linear Induction Pump (ALIP) is required to drive the liquid metal working fluid. The ALIP is an electromagnetic pump that generates a pressure rise through the interaction of a traveling magnetic field produced in the stator and the current flow induced in the liquid metal contained within the pump. Two pump choices were readily available, which included the TDU ALIP and the FPS ALIP. Both pumps were previously tested individually at NASA MSFC in their ALIP Test Circuit (ATC) ${ }^{1}$. These tests produced pump curves and performance maps which defined the expected pump performance. Reactor Simulator testing performed at NASA MSFC was done using the TDU ALIP, which was not capable of providing the nominal pressure and flow rate due to a manufacturing irregularity. As a result, the tests of the Reactor Simulator subsystem (shown in Figure 1) were repeated at GRC, for the FPS ALIP. To help anticipate the pressure demand of the Reactor Simulator loop, computational predictions were generated as part of the process to validate the FPS ALIP's ability to provide the nominal flow.

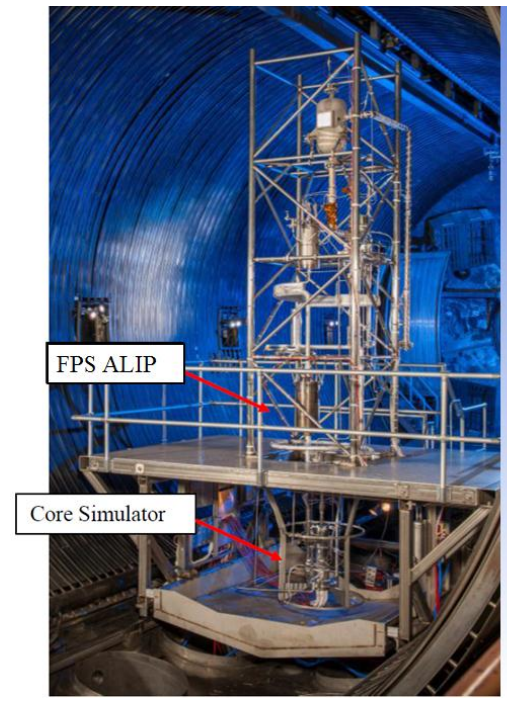

(a) Test article

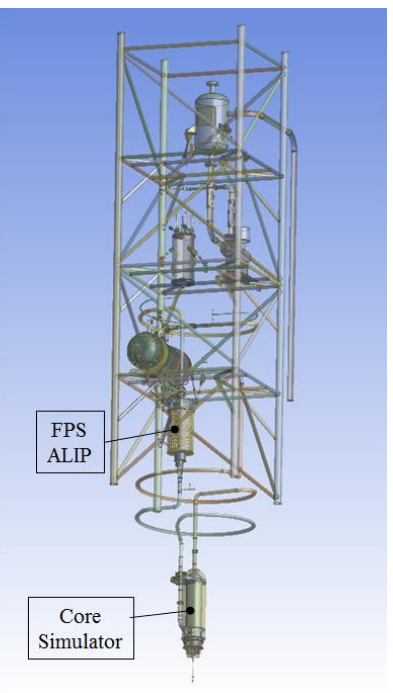

(b) CAD Model

\section{Figure 1. Fission Power System (FPS) Technology Demonstration Unit (TDU).}

The CAD (Computer-Aided-Design) model of the TDU was used to generate the computational model. The FPSALIP and the HXM were removed from the model, and a wedge flow meter was added. This configuration is the same as the hardware that was ultimately tested. The components of the full-scale TDU are shown in Figure 2. The computational model of the NaK loop only is shown in Figure 3. The FPS ALIP was removed so that the inlet and exit boundary conditions could be applied at that location. Calculated pressure drops represent ALIP pump requirements. The primary components in this configuration are the flow meter, tubing, and the core simulator. 


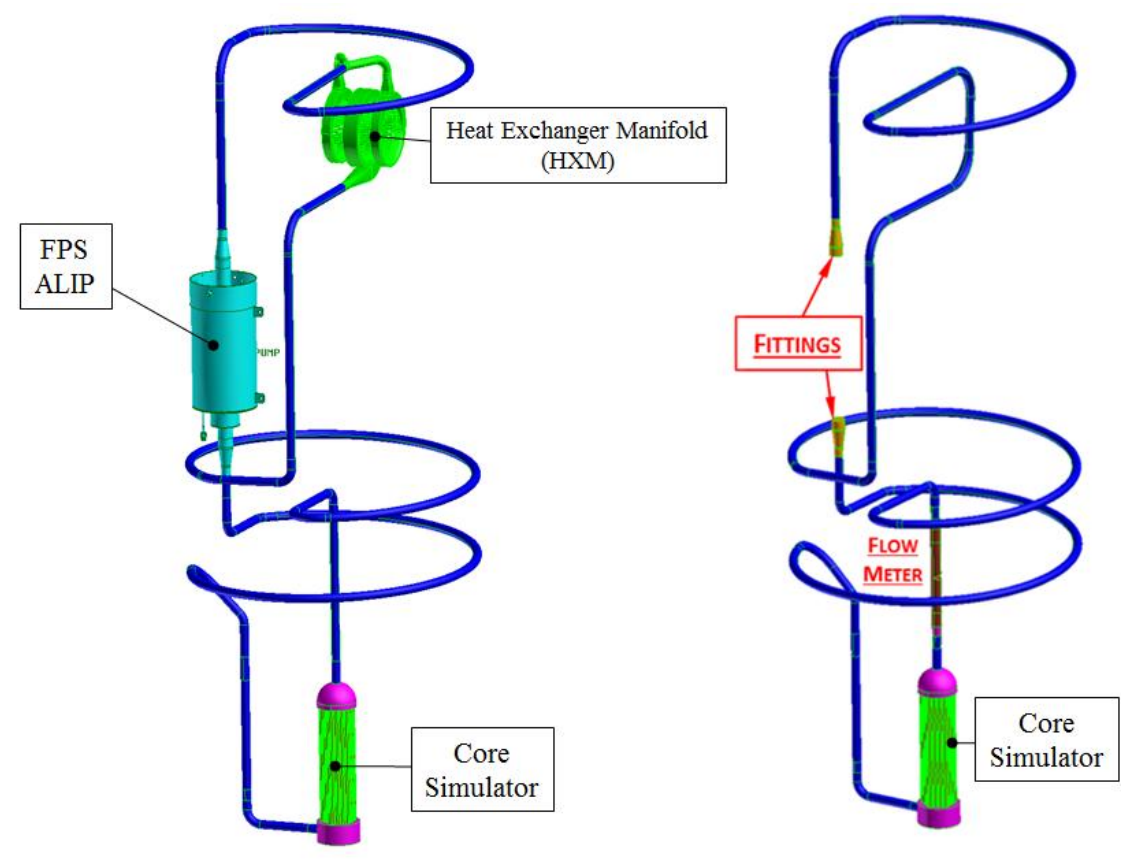

(a) Original CAD Model

(b) Trimmed CAD Model

Figure 2. The RxSim Subsystem.

\section{Analysis Description}

ANSYS Mesher ${ }^{\mathrm{TM}}$ and ANSYS Fluent ${ }^{\mathrm{TM}}$ were used to generate the mesh and calculate solutions. The generated unstructured mesh has approximately 2.5 million cells. Boundary conditions include a mass flow inlet, a pressure outlet, and no-slip walls. The inlet temperature was held constant for all cases at $798 \mathrm{~K}$, although this isn't expected to be critical at this time since all walls have an adiabatic boundary condition. For future cases, heat flux boundary conditions will be added to the Core Simulator (to add heat), and to the HXM (to remove heat). The focus of this series of calculations is to predict the pressure losses throughout the loop and the subsequent requirement for the ALIP over a range of mass flows.

Numerical solutions were obtained for the single-phase Navier-Stokes equations. Temperature-dependent material properties for liquid metal (NaK - Sodium Potassium) were used for the fluid. The Semi-Implicit Method for Pressure Linked Equations (SIMPLE) ${ }^{2}$ algorithm was used for the pressure-velocity coupling. Convective terms were discretized using a second-order upwind scheme. Spatial gradients are computed using a cell based least squares method. Steady-state simulations were conducted until the root mean square (rms) values of the continuity, velocity, and energy residuals were reduced by at least four orders of magnitude. Calculations through a pre-determined mass flow range were performed using both the Spalart-Allmaras ${ }^{3}$ and the Realizeable $\mathrm{k}-\varepsilon^{4}$ turbulence models.

The Spalart-Allmaras model is a one-equation model that solves a modeled transport equation for the kinetic eddy (turbulent) viscosity. It is not calibrated for general industrial flows and does produce relatively larger errors for some free shear flows, especially plane and round jet flows. Because it is a one-equation model, it uses less resources (CPU time) than the other two-equation models. However, it cannot be relied on to predict the decay of homogeneous, isotropic turbulence.

The Realizeable k- $\varepsilon$ model is a two-equation model. It differs from the standard k- $\varepsilon$ model by using an alternative formulation for the turbulent viscosity, and by using a modified transport equation for the dissipation rate $\varepsilon$, which has been derived from an exact equation for the transport of the mean-square vorticity fluctuation. The term "realizeable" means that the model satisfies certain mathematical constraints on the Reynolds stresses, consistent with the physics of turbulent flows. The model has been extensively validated for a wide range of flows, which includes free flows including jets and mixing layers, channel and boundary layer flows, and separated flows. This model uses considerably more CPU resources than the Spalart-Allmaras model while obtaining a higher degree of accuracy.

American Institute of Aeronautics and Astronautics 


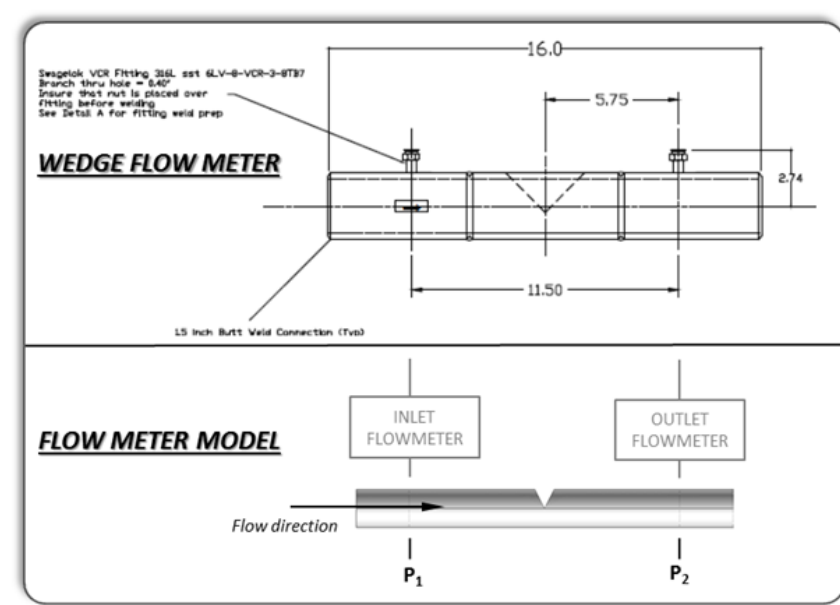

(a) Geometry of wedge flow meter

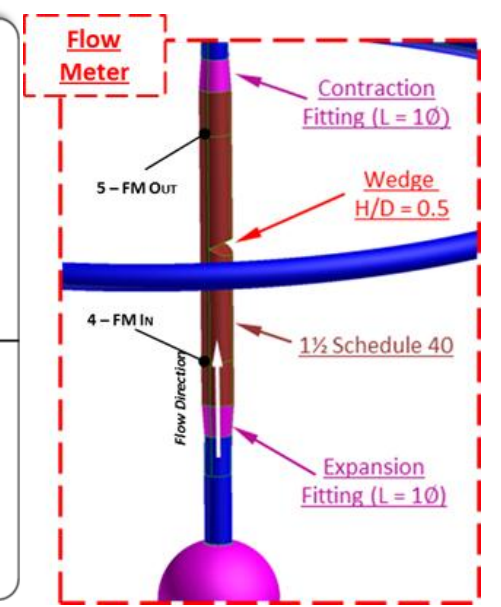

(b) Flow meter in model

Figure 3. The Wedge Flow Meter.

\section{A. Wedge Flow Meter}

The wedge flow meter was manufactured by ABB. The geometry and the location of this flow meter is shown in Figure 3. The flow meter in the model is located just downstream of the core simulator. The wedge flow meter is essentially a $1 \frac{1 / 2}{2}$ inch carbon steel Schedule 40 pipe with a wedge inserted in the pipe and the walls roughened. The pipe roughness of the wedge flow meter is $0.00015 \mathrm{ft}(0.00004572 \mathrm{~m})$. The wedge and the roughened flow meter walls are designed to allow us to take advantage of our knowledge of turbulence to predict the mass flow through observed pressure drops within the flow meter. When the flow meter was shipped, it included numerical predictions performed by ABB using a computational solver called ABB Suites. When the model was generated (at NASA GRC), these calculations were repeated using both OpenFOAM and ANSYS Fluent ${ }^{\mathrm{TM}}$. The results of these simulations are shown in Figure 4.

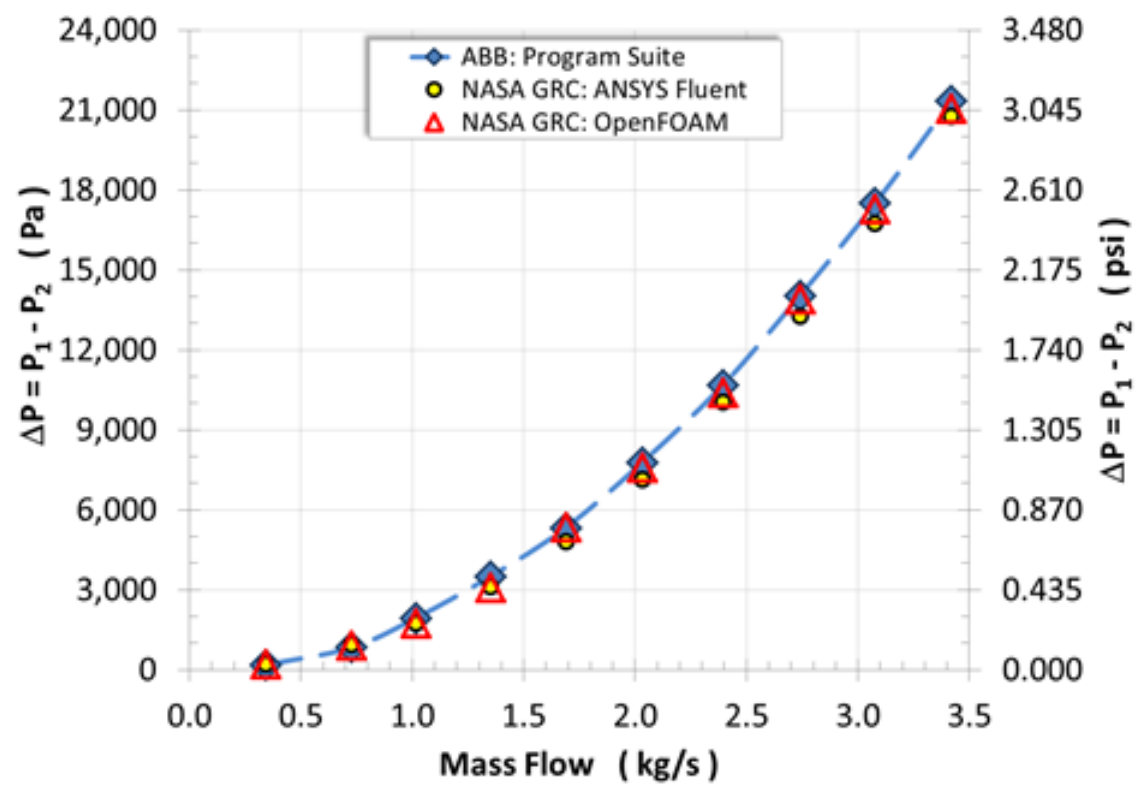

Figure 4. Predicted pressure drops in the wedge flow meter. 


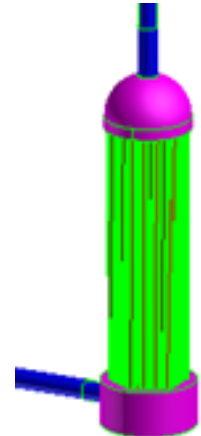

(a) CAD model of Core Simulator

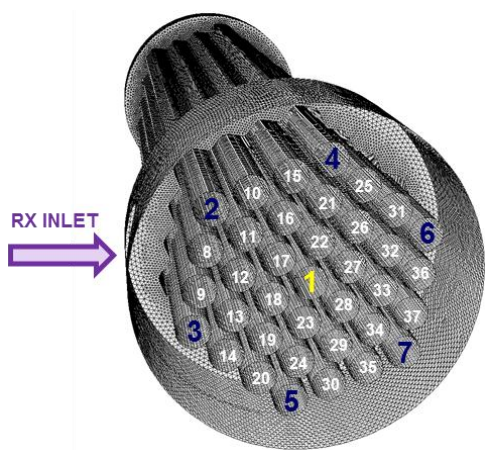

(b) Mesh of Core Simulator

Figure 5. The Core Simulator.

\section{B. The Core Simulator}

In future simulations, the core simulator will be used to heat the working fluid. In the current series of calculations the focal point is pressure drop. Efforts were made to include as many geometric details of the core simulator as possible, because they will influence the pressure drop. The geometry of the Core Simulator is shown in Figures 5 and 6 . The Core Simulator contains 37 rods, which collectively provides a reduced flow area for the flow entering and exiting. The primary flow path through the core simulator can be seen in Figure 6, in the leftmost image. The surfaces within this simulator are considered to be smooth.

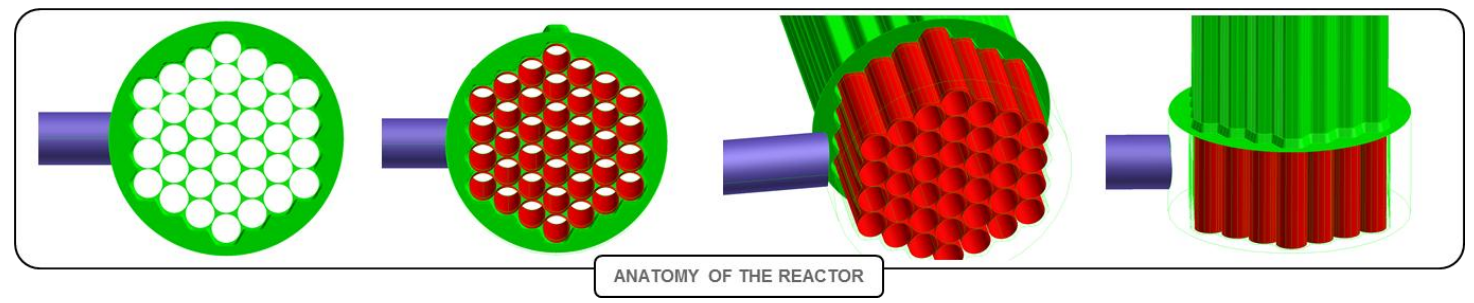

Figure 6. Anatomy of the Core Simulator.

\section{The ALIP}

As mentioned before, the ALIP is an electromagnetic pump that generates a pressure rise through the interaction of a traveling magnetic field produced in the stator and the current flow induced in the liquid metal contained within the pump. Two pump choices were available, which includes the TDU ALIP (Figure 7a) and the FPS ALIP (Figure 7b). The target nominal flow is $1.75 \mathrm{~kg} / \mathrm{sec}$. Looking at the performance curves for the TDU ALIP shown Figure 7a, it is clear that the TDU ALIP does not have the capacity to produce the desired nominal flow. However, the performance curves for the FPS ALIP (shown in Figure 7b) suggests that this pump may be able to produce the required flow at nominal conditions, but its ability to achieve the nominal flow is based on the pressure losses in the RxSim subsystem.

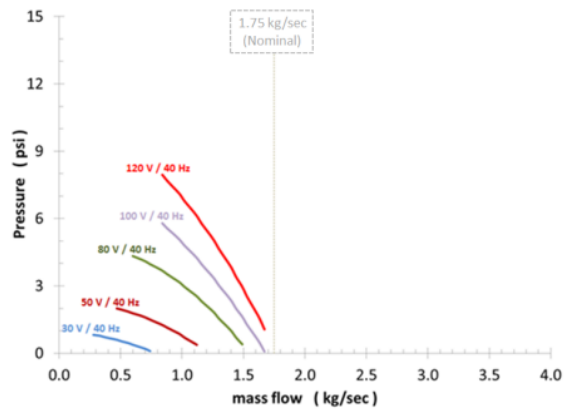

(a) TDU ALIP

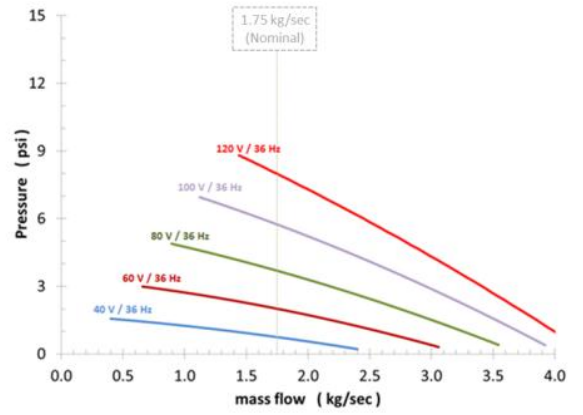

(b) FPS ALIP

Figure 7. Measured performance curves of the ALIP. (Courtesy of NASA MSFC). 


\section{Results}

The calculated solutions of the RxSim subsystem are shown in Figure 8 (using the Spalart-Allmaras turbulence). Mass flow boundary conditions were applied to the face at Station 0 (inlet) and pressure outlet boundary conditions applied to the face on Station 9 (outlet), which corresponds to the location of the ALIP. The schematic in Figure 8 shows the location of each station, while the plot in Figure 8 shows the pressure at each station. The plot in Figure 8 can be used to calculate the pressure drop across each component in the loop for various inlet mass flow settings. A plot was also generated using the results from the Realizeable k- $\varepsilon$ turbulence model, but is not shown here since the pressure drop on a component level are nearly indistinguishable in a plot. Instead, the total differences between Station 9 and Station 0 (the entire loop) are shown for both the Spalart-Allmaras and the Realizeable k- $\varepsilon$ turbulence models in Figure 9. The green circles represent the measured pressure drops during laboratory testing.

Curves fits were generated for the results of Figure 9, and are shown below. Note that all curve fits have a zero yaxis intercept, which corresponds to 0 pressure loss when there is zero mass flow. The predictions using the SpalartAllmaras and Realizeable k- $\varepsilon$ turbulence models were generated at the same mass flow increment (from 0 to 3.0 at increments of 0.5), but the actual measured data was not. As a result, the resulting curve fits are as follows:

$$
\begin{aligned}
\Delta P=1.01187(m f)^{2}+0.358372(m f)-0.0 & \text { for the measured data } \\
\Delta P=1.28385(m f)^{2}+0.405565(m f)-0.0 & \text { for predictions using S-A model } \\
\Delta P=1.10399(m f)^{2}+0.507516(m f)-0.0 & \text { for predictions using k- } \varepsilon \text { model }
\end{aligned}
$$

The curve-fit pressure loss predictions produced by the various turbulence models are compared to the curve-fit of the measured data, and their differences can be seen in Figure 9. This plot indicates that at a mass flow of $2.5 \mathrm{~kg} / \mathrm{s}$, the S-A turbulence model prediction was 1.827 psi higher than measured data while at the same mass flow, the Realizeable k- $\varepsilon$ turbulence model was 0.964 psi higher than measured data.

Another use for these equations is to estimate the mass flow and anticipated pressure the ALIP can provide. This is done by defining a curve-fit equation for the ALIP operating at $120 \mathrm{~V}$ and $36 \mathrm{~Hz}$ (which is the highest performing ALIP condition tested, see Figure 9). The equation at these operating conditions are

$$
\Delta P=-0.1760963(m f)^{2}-2.09680(m f)+12.19200 \text { for the FPS ALIP at } 120 \mathrm{~V} / 36 \mathrm{~Hz}
$$

At this point, the pump equation curve-fit can be simultaneously solved with each of the above pressure loss curvefits (one at a time), which will provide a mass flow where these curves intersect. The intersection point represents the peak mass flow available from the ALIP (based on the predicted and measured results) while connected to the RxSim loop.
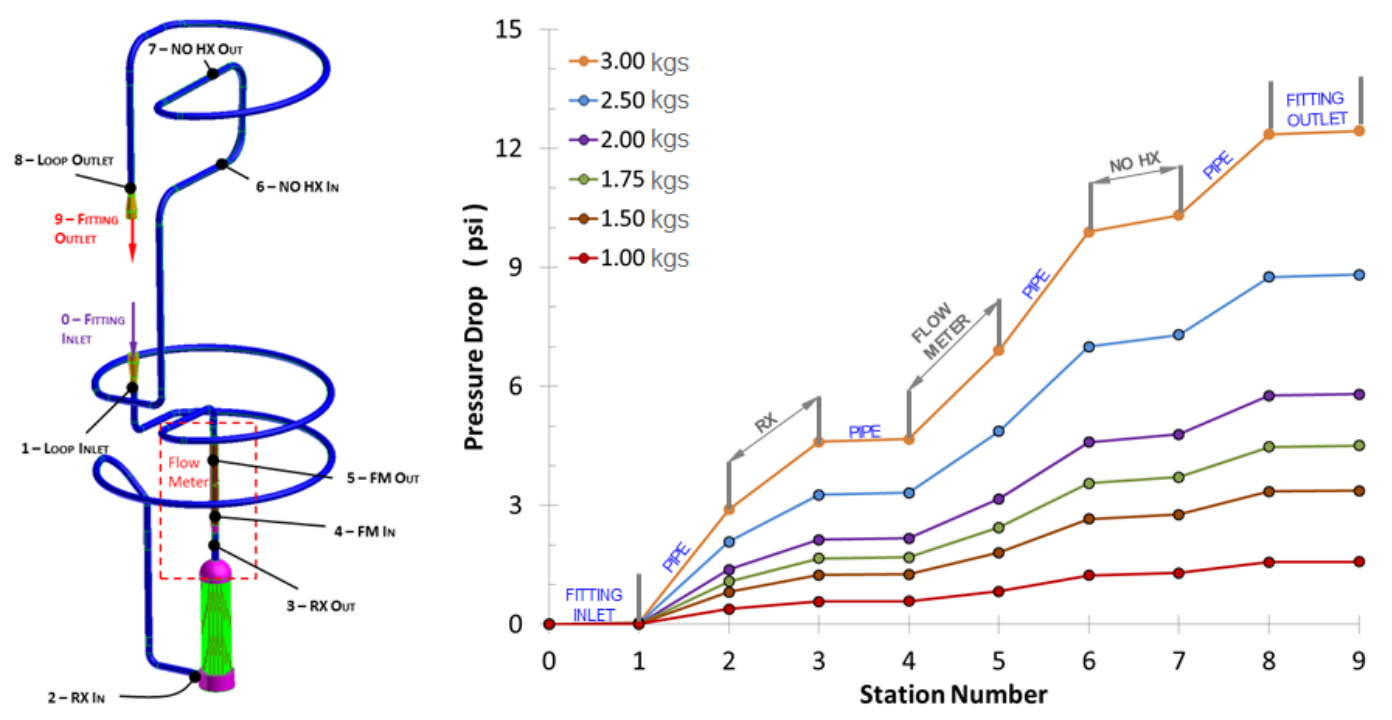

Figure 8. Pressure loss predictions of RxSim subsystem.

American Institute of Aeronautics and Astronautics 


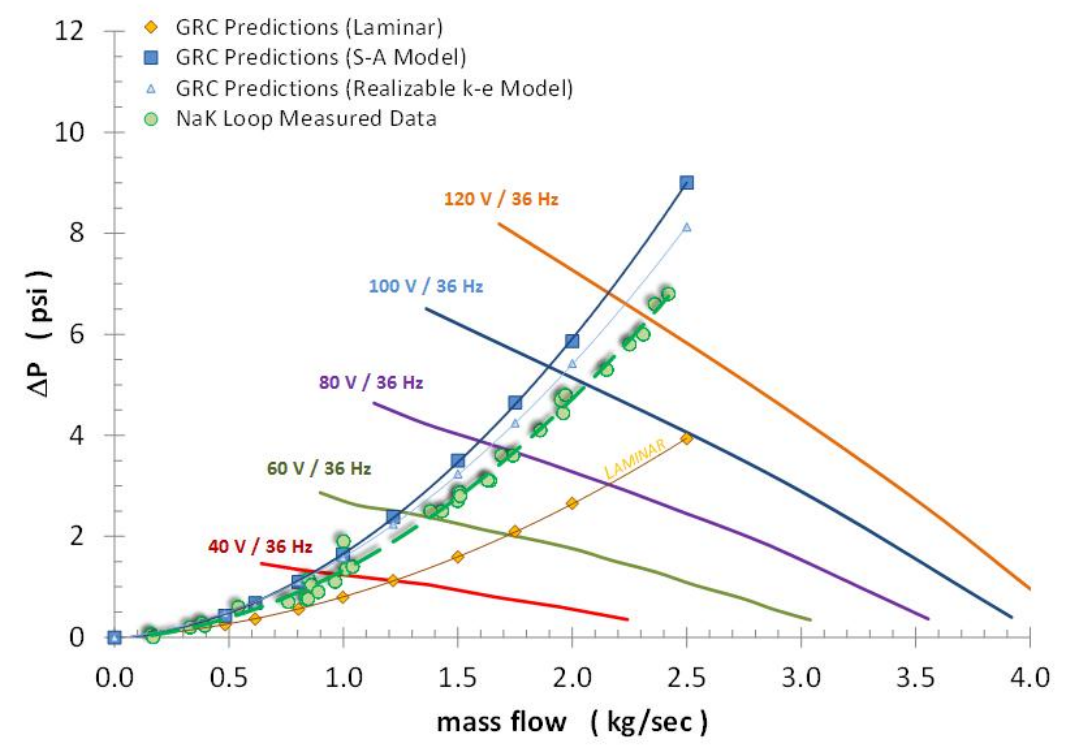

Figure 9. Predicted loop pressure losses in RxSim subsystem.

To estimate the peak mass flow available from the FPS ALIP based on measured data:

$$
\begin{gathered}
P_{M E A S}=P_{P U M P} \\
1.01187 m f^{2}+0.358372 m f-0.0=-0.1760963 m f^{2}-2.0968 m f+12.192 \\
1.187966 m f^{2}+2.455172 m f-12.192=0 \\
m f^{2}+2.066702 m f-10.26292=0
\end{gathered}
$$

which results in an available mass flow $=2.333 \mathbf{~ k g} / \mathbf{s e c}$. This is the maximum mass flow that the FPS ALIP was able to supply to the RxSim loop during laboratory testing, which is enough to provide flow at the nominal conditions.

\section{Conclusions}

GRC Laboratory testing of the RxSim loop with the FPS ALIP at the maximum flow was $2.333 \mathrm{~kg} / \mathrm{sec}$. Computational predictions of the RxSim loop were generated, and the results were curve-fitted to produce an equation that describes pressure loss as a function of mass flow. This was done using two different turbulence models (SpalartAllmaras and Realizeable k- $\varepsilon$ ). When using predictions from the S-A turbulence model, the predicted available mass flow $=2.157 \mathrm{~kg} / \mathrm{sec}$. Based on measured data, this corresponds to a mass flow error of $-0.173 \mathrm{~kg} / \mathrm{sec}(-7.4 \%)$ when using the S-A turbulence model.

If the same calculation is done using predictions from the Realizeable k- $\varepsilon$ turbulence model, then the predicted available mass flow $=2.223 \mathrm{~kg} / \mathrm{sec}$. Based on measured data, this corresponds to a mass flow error of $-0.110 \mathrm{~kg} / \mathrm{sec}$ $(-4.7 \%)$ when using the Realizeable k- $\varepsilon$ turbulence model.

The combination of laboratory measurements and computational simulations indicate that the FPS ALIP can provide adequate pumping for the TDU.

\section{Acknowledgments}

The author would like to thank the Space Technology Mission Directorate and the Game-Changing Development Program Office.

\section{References}

${ }^{1}$ Polzin, K.A., Pearson, J.B., Schoenfeld, M.P., Webster, K., Houts, M.G., Godfroy, T.J. and Bossard, J.A., "Performance Testing of a Prototypic Annular Linear Induction Pump for Fission Surface Power", NASA/TP-2010216430, 2010.

American Institute of Aeronautics and Astronautics 
${ }^{2}$ Vandoormaal, J. P., and Raithby, G. D.,"'Enhancements of the SIMPLE Method for Predicting Incompressible Fluid Flows", Numerical Heat Transfer 7, pp 147-163, 1984.

${ }^{3}$ Spalart, P. and Allmaras, S., "A one-equation turbulence model for aerodynamic flows", Technical Report AIAA92-0439, American Institute of Aeronautics and Astronautics, 1992.

${ }^{4}$ Shih, T.-H., Liou, W. W., Shabbir, A. Yang, Z., and Zhu, J., "A New k-e Eddy-Viscosity Model for High Reynolds Number Turbulent Flows - Model Development and Validation”, NASA TM 106721, 1994. 